PROCEEDINGS OF THE

AMERICAN MATHEMATICAL SOCIETY

Volume 124, Number 12, December 1996, Pages 3619-3625

S 0002-9939(96)03501-0

\title{
LOCALLY SOLVABLE FACTORS OF VARIETIES
}

\author{
KEITH A. KEARNES
}

(Communicated by Lance W. Small)

\begin{abstract}
We give necessary and sufficient local conditions, which are easy to check, for a locally finite variety $\mathcal{V}$ to decompose as the product of a locally solvable subvariety $\mathcal{U}$ and a subvariety $\mathcal{W}$ which has type set disjoint from the type set of $\mathcal{U}$.
\end{abstract}

\section{INTRODUCTION}

A variety is an equationally definable class of similar algebras. The category of varieties under interpretation has finite products, so given varieties $\mathcal{U}$ and $\mathcal{W}$ one can form $\mathcal{V}=\mathcal{U} \times \mathcal{W}$. This product of $\mathcal{U}$ and $\mathcal{W}$ is sometimes called the varietal product, the varietal sum, the direct sum or the independent join of $\mathcal{U}$ and $\mathcal{V}$, and the product symbol is sometimes $\times, \otimes$, or $\oplus$. We call it simply the product and we use $\times$. We remark that the equality $\mathcal{V}=\mathcal{U} \times \mathcal{W}$ only determines $\mathcal{V}$ up to term equivalence (which means isomorphism in the category of varieties), and this equality implies that $\mathcal{U}$ and $\mathcal{W}$ are term equivalent to subvarieties of $\mathcal{V}$.

There are several well-known examples of varieties which are products of subvarieties. The variety $\mathcal{R B}$ of rectangular bands is $\mathcal{L Z} \times \mathcal{R Z}$ where $\mathcal{L} \mathcal{Z}$ is the variety of left-zero semigroups and $\mathcal{R} \mathcal{Z}$ is the variety of right-zero semigroups. If $\mathbf{R}$ and $\mathbf{S}$ are unital rings, then for the corresponding module varieties we have

$$
\mathcal{M}_{\mathbf{R} \times \mathbf{S}}=\mathcal{M}_{\mathbf{R}} \times \mathcal{M}_{\mathbf{S}}
$$

One can generalize the latter example. If $\mathcal{V}$ is any variety whose members have an underlying group structure and $\mathcal{V}$ is generated by $\mathcal{U} \cup \mathcal{W}$ where $\mathcal{U}$ and $\mathcal{W}$ are subvarieties of $\mathcal{V}$ which have trivial intersection, then $\mathcal{V}=\mathcal{U} \times \mathcal{W}$.

When $\mathcal{V}=\mathcal{U} \times \mathcal{W}$, then every member of $\mathcal{V}$ has a canonical factorization as a product of a member of $\mathcal{U}$ and a member of $\mathcal{W}$, and every homomorphism between members of $\mathcal{V}$ respects these canonical factorizations. Thus, it is straightforward to reduce virtually any question about $\mathcal{V}$ to the corresponding question for each factor variety. It is precisely this fact that makes the recognition of such factorizations of varieties desirable. One of the deepest theorems in all of general algebra is the theorem of R. McKenzie and M. Valeriote in [5] which states that if $\mathcal{V}$ is a locally finite variety which has a decidable first-order theory, then $\mathcal{V}$ decomposes as

$$
\mathcal{S} \times \mathcal{A} \times \mathcal{D}
$$

Received by the editors September 7, 1994 and, in revised form, June 5, 1995.

1991 Mathematics Subject Classification. Primary 08B25; Secondary 08A05.

Research supported by a fellowship from the Alexander von Humboldt Stiftung.

(C)1996 American Mathematical Society 
where $\mathcal{S}$ is a decidable multisorted unary variety, $\mathcal{A}$ is a decidable affine variety, and $\mathcal{D}$ is a decidable discriminator variety. Based on this factorization, they have described an algorithm which converts a finite algebra $\mathbf{A}$ in a finite language into a finite ring $\mathbf{R}$ such that $\mathcal{V}(\mathbf{A})$ is decidable if and only if the variety of $\mathbf{R}$-modules is decidable. The question of which finite rings have a decidable theory of modules is still open.

The McKenzie-Valeriote proof uses tame congruence theory, the viewpoint of which is that every finite algebra may be approximated locally by algebras of five very different, but classifiable "types". An extremely valuable technique, developed by Valeriote in his Ph. D. thesis, [6], is that establishing "transfer principles" shows that the approximating algebras of different types are essentially independent of each other. This is a basic first step towards decomposing a locally finite variety into a product of subvarieties of disjoint type sets; and this approach is especially well tailored to the techniques of tame congruence theory. The following question arises: After establishing that the necessary transfer principles hold, what else must be checked to guarantee a product decomposition? This question was answered in Section 3 of [1] in the sense that a simple list of necessary and sufficient conditions were given for such decompositions to exist. In this paper, we show that direct decompositions occur more readily when a proposed direct factor is locally solvable. Our results eliminate the need for some of the semantic embeddings in the McKenzie-Valeriote proof. In particular, all of Chapter 13 of [5] can be eliminated.

Our notation is standard with one exception: we denote the operations of a lattice by + for join and juxtaposition for meet. For the congruence lattice of $\mathbf{A}$, we write $0^{\mathbf{A}}$ and $1^{\mathbf{A}}$ for the least and largest elements, although we may drop the superscripts. Our notation for product congruences follows [2] and we describe it briefly now. Let $\pi_{i}: \mathbf{A}_{0} \times \mathbf{A}_{1} \rightarrow \mathbf{A}_{i}, i=0,1$, denote the canonical projection onto the $i^{\text {th }}$ factor. If $\alpha \in \operatorname{Con}\left(\mathbf{A}_{i}\right)$, then $\alpha_{i}$ denotes the congruence $\pi_{i}^{-1}(\alpha)$ of $\mathbf{A}_{0} \times \mathbf{A}_{1}$ except in the case where $\alpha=0$; in this case we write $\eta_{i}$ for $\pi_{i}^{-1}(\alpha)=\pi_{i}^{-1}(0)$, which is the kernel of the projection onto the $i^{\text {th }}$ factor algebra. If $\alpha \in \operatorname{Con}\left(\mathbf{A}_{0}\right)$ and $\beta \in \operatorname{Con}\left(\mathbf{A}_{1}\right)$; then, according to our rules, the meet $\alpha_{0} \beta_{1}$ is the congruence on $\mathbf{A}_{0} \times \mathbf{A}_{1}$ consisting of the pairs

$$
\left\{\langle(x, y),(u, v)\rangle \in\left(A_{0} \times A_{1}\right)^{2} \mid(x, u) \in \alpha,(y, v) \in \beta\right\} .
$$

Congruences of this form are called product congruences. A fact about product congruences which we shall use without comment in this paper is that two product congruences which permute coordinatewise must permute.

\section{Splitting OfF LOCALly SOlvable FaCtors}

The book [3] develops a local structure theory for finite algebras. For a given finite algebra $\mathbf{A}$, the local structure is partially encoded in a labeling of the congruence lattice of $\mathbf{A}$. In this paper we will not need to know anything of this theory except that whenever $\alpha$ is covered by $\beta$ in $\mathbf{C o n}(\mathbf{A})$ it is possible to assign a label to this covering pair. This label, written typ $(\alpha, \beta)$ and called the type of $\langle\alpha, \beta\rangle$, is some number from the set $\{\mathbf{1}, \mathbf{2}, \mathbf{3}, \mathbf{4}, \mathbf{5}\}$. One property about allowable labelings which we will need is the fact that perspective covering pairs must have the same label. What this means is that if $\alpha_{1} \prec \beta_{1}, \alpha_{2} \prec \beta_{2}, \alpha_{2} \vee \beta_{1}=\beta_{2}$, and $\alpha_{2} \wedge \beta_{1}=\alpha_{1}$, then typ $\left(\alpha_{1}, \beta_{1}\right)=\operatorname{typ}\left(\alpha_{2}, \beta_{2}\right)$. 
Definition 2.1. Let $T, T^{\prime} \subseteq\{\mathbf{1}, \mathbf{2}, \mathbf{3}, \mathbf{4}, \mathbf{5}\}$ be sets of type labels. A finite algebra $\mathbf{A}$ is said to satisfy the $\left\langle T, T^{\prime}\right\rangle$ transfer principle if there do not exist congruences $\alpha \prec \beta \prec \gamma$ on $\mathbf{A}$ such that

(1) typ $(\alpha, \beta) \in T$ and typ $(\beta, \gamma) \in T^{\prime}$, and

(2) the interval $I[\alpha, \gamma]$ in $\mathbf{C o n}(\mathbf{A})$ is $\{\alpha, \beta, \gamma\}$.

A locally finite variety is said to satisfy the $\left\langle T, T^{\prime}\right\rangle$ transfer principle if its finite members do.

If $\mathbf{A}$ is a finite algebra and $T$ is a set of type labels, then we can define a binary relation $\underset{T}{\widetilde{T}}$ on $\mathbf{C o n}(\mathbf{A})$ by specifying that

$$
\alpha \underset{T}{\alpha} \beta \Leftrightarrow \operatorname{typ}\{\alpha \beta, \alpha+\beta\} \subseteq T .
$$

A basic result connecting these relations to the transfer principles is Theorem 3.10 of [1], which proves the following.

Theorem 2.2. Let $\mathbf{A}$ be a finite algebra and assume that $T$ and $T^{\prime}$ are disjoint sets of type labels such that typ $\{\mathbf{A}\} \subseteq T \cup T^{\prime}$. Then $\mathbf{A}$ satisfies the $\left\langle T, T^{\prime}\right\rangle$ and $\left\langle T^{\prime}, T\right\rangle$ transfer principles if and only if $\underset{T}{\sim}$ and $\underset{T^{\prime}}{\sim}$ are permuting congruences on $\operatorname{Con}(\mathbf{A})$.

Under the assumptions of the above theorem, let $\rho_{T}\left(\rho_{T^{\prime}}\right)$ denote the largest congruences $\underset{T}{\sim}$-related $\left(\underset{T^{\prime}}{\sim}-\right.$ related $)$ to $0^{\mathbf{A}}$. The congruence $\rho_{T}\left(\rho_{T^{\prime}}\right)$ is called the $T$-radical ( $T^{\prime}$-radical) of A. Theorem 2.2 proves that when $\mathbf{A}$ satisfies the $\left\langle T, T^{\prime}\right\rangle$ and $\left\langle T^{\prime}, T\right\rangle$ transfer principles, then $\mathbf{C o n}(\mathbf{A})$ is naturally isomorphic to the direct product

$$
I\left[\rho_{T}, 1^{\mathbf{A}}\right] \times I\left[\rho_{T^{\prime}}, 1^{\mathbf{A}}\right]
$$

considered as labeled lattices. In fact, $\rho_{T}$ and $\rho_{T^{\prime}}$ are complements in $\mathbf{C o n}(\mathbf{A})$ and the natural homomorphism from $\mathbf{A}$ into $\left(\mathbf{A} / \rho_{T}\right) \times\left(\mathbf{A} / \rho_{T^{\prime}}\right)$ is a subdirect embedding. A direct consequence of Theorem 2.2 is the following.

Corollary 2.3. Assume that $\mathbf{A}$ is finite, and that every subalgebra of $\mathbf{A}^{2}$ satisfies the $\left\langle T, T^{\prime}\right\rangle$ and $\left\langle T^{\prime}, T\right\rangle$ transfer principles. If $\mathbf{A}$ is a subdirect product of $\mathbf{B}$ and $\mathbf{C}$, where typ $\{\mathbf{B}\} \subseteq T^{\prime}$ and typ $\{\mathbf{C}\} \subseteq T$, then the $T$-radical and $T^{\prime}$-radical of $\mathbf{A}$ are $\left.\eta_{0}\right|_{A}$ and $\left.\eta_{1}\right|_{A}$, respectively, and every congruence on $\mathbf{A}$ is the restriction of a product congruence on $\mathbf{B} \times \mathbf{C}$.

In this corollary, the assumption that every subalgebra of $\mathbf{A}^{2}$ satisfies the transfer principles implies that $\mathbf{H S}\left(\mathbf{A}^{2}\right)$ satisfies the transfer principles, since homomorphic images inherit the transfer principles. The hypothesis is only used to force $\mathbf{B} \times \mathbf{C}$, which is in $\mathbf{H S}\left(\mathbf{A}^{2}\right)$, to satisfy the transfer principles.

The next result we need is Theorem 2.1 of [4].

Theorem 2.4. Let $\mathbf{A}$ be a finite algebra, let $B$ be a maximal subuniverse of $\mathbf{A}$, and let $\gamma$ be a minimal abelian congruence on $\mathbf{A}$. If $\left.\gamma\right|_{B}>0$, then $B$ is a union of $\gamma$-classes.

In this theorem, the statement that $\gamma$ is a minimal abelian congruence on $\mathbf{A}$ means that $\operatorname{typ}\left(0^{\mathbf{A}}, \gamma\right) \in\{\mathbf{1}, \mathbf{2}\}$. In what follows, the phrase that "typ $\{\mathcal{V}\} \subseteq$ $\{\mathbf{1}, \mathbf{2}\}$ " is equivalent to " $\mathcal{V}$ is locally solvable". 
Theorem 2.5. Let $\mathbf{A}$ be a finite algebra and let $T$ and $T^{\prime}$ be sets of type labels for which

(1) $T \cap T^{\prime}=\emptyset$,

(2) $\operatorname{typ}\{\mathbf{A}\} \subseteq T \cup T^{\prime}$, and

(3) $T \subseteq\{\mathbf{1}, \mathbf{2}\}$.

If the $\left\langle T, T^{\prime}\right\rangle$ and $\left\langle T^{\prime}, T\right\rangle$ transfer principles hold in all subalgebras of $\mathbf{A}^{2}$, then

$$
\mathbf{A} \cong\left(\mathbf{A} / \rho_{T}\right) \times\left(\mathbf{A} / \rho_{T^{\prime}}\right)
$$

Proof. We assume that the theorem is false and argue to a contradiction. To simplify notation, we will write $\mathbf{A}_{0}$ for $\mathbf{A} / \rho_{T}$ and $\mathbf{A}_{1}$ for $\mathbf{A} / \rho_{T^{\prime}}$. Remember, though, that typ $\left\{\mathbf{A}_{0}\right\}=\operatorname{typ}\left\{\rho_{T}, 1^{\mathbf{A}}\right\} \subseteq T^{\prime}$ and similarly that typ $\left\{\mathbf{A}_{1}\right\} \subseteq T$. Let $\mathbf{A}$ be a finite algebra which satisfies the hypotheses of the theorem, but for which the natural embedding $\varphi: \mathbf{A} \rightarrow \mathbf{A}_{0} \times \mathbf{A}_{1}$ is not onto. We choose such an $\mathbf{A}$ such that Con $(\mathbf{A})$ has minimum height among all such examples. Identify $\mathbf{A}$ with $\varphi(\mathbf{A})$.

We have assumed that all subalgebras of $\mathbf{A}^{2}$ satisfy the $\left\langle T, T^{\prime}\right\rangle$ and $\left\langle T^{\prime}, T\right\rangle$ transfer principles, which implies that every subalgebra of $\mathbf{A}_{0} \times \mathbf{A}_{1}$ satisfies these transfer principles. For any $\mathbf{B}$ such that $\mathbf{A} \leq \mathbf{B} \leq \mathbf{A}_{0} \times \mathbf{A}_{1}$, we can apply Corollary 2.3 to obtain that $\mathbf{C o n}\left(\mathbf{A}_{0} \times \mathbf{A}_{1}\right) \cong \mathbf{C o n}(\mathbf{B})$ by restriction. If such a $\mathbf{B}$ is a proper subalgebra of $\mathbf{A}_{0} \times \mathbf{A}_{1}$, then $\mathbf{B}$ is subdirectly (but not isomorphically) embedded into

$$
\mathbf{A}_{0} \times \mathbf{A}_{1} \cong\left(\mathbf{B} / \rho_{T}\right) \times\left(\mathbf{B} / \rho_{T^{\prime}}\right) .
$$

In particular, if $\mathbf{B}$ is a proper subalgebra of $\mathbf{A}_{0} \times \mathbf{A}_{1}$ containing $\mathbf{A}$, then $\mathbf{B}$ is also a counterexample to the theorem and the labeled congruence lattice of $\mathbf{B}$ is the same as that of $\mathbf{A}$. By extending $\mathbf{A}$ if necessary, we may assume that $\mathbf{A}$ is a maximal proper subalgebra of $\mathbf{A}_{0} \times \mathbf{A}_{1}$.

Since $\left.\eta_{0}\right|_{A}=\rho_{T}$ and $\left.\eta_{1}\right|_{A}=\rho_{T^{\prime}}$ are complementary congruences of $\mathbf{A}$ which are not a pair of factor congruences, $\rho_{T}$ fails to permute with $\rho_{T^{\prime}}$. Choose $\sigma, \tau \in$ Con $(\mathbf{A})$ such that $0^{\mathbf{A}} \prec \sigma \leq \rho_{T}$ and $0^{\mathbf{A}} \prec \tau \leq \rho_{T^{\prime}}$. We claim that $\sigma$ and $\tau$ fail to permute. Before proving this, let's show that $\sigma$ fails to permute with $\rho_{T^{\prime}}$. If this were not the case, then we would have $\sigma+\rho_{T^{\prime}}=\sigma \circ \rho_{T^{\prime}}$. The algebra $\mathbf{A} / \sigma$ satisfies all the assumptions made about $\mathbf{A}$ and has a congruence lattice of smaller height. By the minimality restriction placed on $\mathbf{A}, \mathbf{A} / \sigma$ has a direct decomposition as described in the theorem. Hence, the $T$-radical of $\mathbf{A} / \sigma$ (which is $\rho_{T} / \sigma$ ) permutes with the $T^{\prime}$-radical (which is $\left(\sigma+\rho_{T^{\prime}}\right) / \sigma$ ). Therefore, $\rho_{T}$ permutes with $\sigma+\rho_{T^{\prime}}=\sigma \circ \rho_{T^{\prime}}$ in $\mathbf{C o n}(\mathbf{A})$. Since $\sigma$ is comparable with $\rho_{T}$ we get that

$$
\begin{aligned}
1^{\mathbf{A}} & =\rho_{T}+\rho_{T^{\prime}} \\
& =\rho_{T}+\sigma+\rho_{T^{\prime}} \\
& =\rho_{T}+\left(\sigma \circ \rho_{T^{\prime}}\right) \\
& =\rho_{T} \circ\left(\sigma \circ \rho_{T^{\prime}}\right) \\
& =\left(\rho_{T} \circ \sigma\right) \circ \rho_{T^{\prime}} \\
& =\rho_{T} \circ \rho_{T^{\prime}},
\end{aligned}
$$

which shows that $\rho_{T}$ permutes with $\rho_{T^{\prime}}$. This contradicts our assumption that $\mathbf{A}$ does not factor as $\left(\mathbf{A} / \rho_{T}\right) \times\left(\mathbf{A} / \rho_{T^{\prime}}\right)$. We conclude that $\sigma$ does not permute with $\rho_{T^{\prime}}$. The argument that $\sigma$ does not permute with $\tau$ is very similar to the one just described. Assume that $\sigma+\tau=\sigma \circ \tau$. By the minimality of $\mathbf{A}$, the algebra $\mathbf{A} / \tau$ satisfies the transfer principles and factors as the product of an algebra of 
type $T$ and an algebra of type $T^{\prime}$. It follows that the $T$-radical of $\mathbf{A} / \tau$ permutes with the $T^{\prime}$-radical. Furthermore, the $T^{\prime}$-radical, which is a coordinate projection kernel, permutes with any product congruence, and therefore permutes with any congruence. Thus, $\rho_{T^{\prime}} / \tau$ permutes with $(\sigma+\tau) / \tau$. This implies that $\rho_{T^{\prime}}$ permutes with $\sigma+\tau=\sigma \circ \tau$, so

$$
\sigma+\rho_{T^{\prime}}=\sigma+\tau+\rho_{T^{\prime}}=(\sigma \circ \tau)+\rho_{T^{\prime}}=\sigma \circ \tau \circ \rho_{T^{\prime}}=\sigma \circ \rho_{T^{\prime}} .
$$

This contradicts our earlier conclusion that $\sigma$ does not permute with $\rho_{T^{\prime}}$, so $\sigma$ cannot permute with $\tau$.

To finish the proof, we will show that $\sigma$ does permute with $\tau$ ! By Corollary 2.3, $\sigma$ is the restriction to $\mathbf{A}$ of a congruence on $\mathbf{A}_{0} \times \mathbf{A}_{1}$ of the form $\eta_{0} \beta_{1}$ where $0 \prec \beta$ in $\mathbf{C o n}\left(\mathbf{A}_{1}\right)$ and $\tau$ is the restriction to $\mathbf{A}$ of a congruence $\alpha_{0} \eta_{1}$ where $0 \prec \alpha$ in $\operatorname{Con}\left(\mathbf{A}_{0}\right)$. Since typ $\left\{\mathbf{A}_{1}\right\} \subseteq T$, and $\operatorname{Con}\left(\mathbf{A}_{0} \times \mathbf{A}_{1}\right)$ is a direct product of labeled lattices, we get that $\operatorname{typ}\left(0, \eta_{0} \beta_{1}\right) \in T \subseteq\{\mathbf{1}, \mathbf{2}\}$. Theorem 2.4 applies to this situation. Since $A$ is a maximal subuniverse of $\mathbf{A}_{0} \times \mathbf{A}_{1}, \eta_{0} \beta_{1}$ is a minimal abelian congruence of $\mathbf{A}_{0} \times \mathbf{A}_{1}$, and $\left.\left(\eta_{0} \beta_{1}\right)\right|_{A}=\sigma>0$, we get that $A$ is a union of $\left(\eta_{0} \beta_{1}\right)$-classes. Choose any pair of elements

$$
(a, b) \in \sigma+\tau=\left.\left(\eta_{0} \beta_{1}+\alpha_{0} \eta_{1}\right)\right|_{A} .
$$

Then, since the product congruences $\eta_{0} \beta_{1}$ and $\alpha_{0} \eta_{1}$ of $\mathbf{A}_{0} \times \mathbf{A}_{1}$ permute, we get that $\left.(a, b) \in\left(\eta_{0} \beta_{1} \circ \alpha_{0} \eta_{1}\right)\right|_{A}$. This means that there is a $c \in \mathbf{A}_{0} \times \mathbf{A}_{1}$ such that $(a, c) \in \eta_{0} \beta_{1}$ and $(c, b) \in \alpha_{0} \eta_{1}$. But the facts that $a \in A,(a, c) \in \eta_{0} \beta_{1}$, and $A$ is a union of $\eta_{0} \beta_{1}$-classes imply that $c \in A$. We get that $\left.(a, c) \in\left(\eta_{0} \beta_{1}\right)\right|_{A}$ and $\left.(c, b) \in\left(\alpha_{0} \eta_{1}\right)\right|_{A}$. Therefore, $\left.\left.(a, b) \in\left(\eta_{0} \beta_{1}\right)\right|_{A} \circ\left(\alpha_{0} \eta_{1}\right)\right|_{A}=\sigma \circ \tau$. The pair $(a, b) \in \sigma+\tau$ was chosen arbitrarily, so $\sigma+\tau=\sigma \circ \tau$ and $\sigma$ and $\tau$ permute. This contradiction finishes the proof.

Corollary 2.6. Let $\mathcal{V}$ be a locally finite variety and let $T_{1}=\{\mathbf{1}\}, T_{2}=\{\mathbf{2}\}$ and $T_{3} \subseteq\{\mathbf{3}, \mathbf{4}, \mathbf{5}\}$. Assume that typ $\{\mathcal{V}\} \subseteq T_{1} \cup T_{2} \cup T_{3}$. If $\mathcal{V}$ satisfies the $\left\langle T_{i}, T_{j}\right\rangle-$ transfer principle for each $i \neq j$, then each finite member of $\mathcal{V}$ is isomorphic to a direct product $\mathbf{A}_{1} \times \mathbf{A}_{2} \times \mathbf{A}_{3}$ where typ $\left\{\mathbf{A}_{i}\right\} \subseteq T_{i}$.

Proof. Choose any finite $\mathbf{A} \in \mathcal{V}$ and apply Theorem 2.5 to $\mathbf{A}$ with $T=T_{1} \cup T_{2}$ and $T^{\prime}=T_{3}$. This proves that $\mathbf{A} \cong \mathbf{B} \times \mathbf{A}_{3}$, where typ $\{\mathbf{B}\} \subseteq T_{1} \cup T_{2}$ and typ $\left\{\mathbf{A}_{3}\right\} \subseteq T_{3}$. Then apply Theorem 2.5 to $\mathbf{B}$ to decompose it into $\mathbf{A}_{1} \times \mathbf{A}_{2}$, where typ $\left\{\mathbf{A}_{i}\right\} \subseteq T_{i}$.

In [1], a relation of the form $\underset{T}{\sim}$ is said to be hereditary throughout $\mathcal{V}$ if whenever $\mathbf{A} \in \mathcal{V}$ is finite and $\mathbf{B} \leq \mathbf{A}$ is a subalgebra, we have

$$
\left.\left.\alpha \underset{T}{\sim} \beta \Rightarrow \alpha\right|_{B} \underset{T}{\sim} \beta\right|_{B}
$$

for any two congruences $\alpha, \beta \in \operatorname{Con}(\mathbf{A})$. If there exists a decomposition $\mathcal{V}=$ $\mathcal{V}_{T} \times \mathcal{V}_{T^{\prime}}$ where $T$ and $T^{\prime}$ are disjoint sets of type labels and typ $\left\{\mathcal{V}_{X}\right\} \subseteq X$, then clearly $\underset{T}{\sim}$ and $\underset{T^{\prime}}{\sim}$ are hereditary and the $\left\langle T, T^{\prime}\right\rangle$ and $\left\langle T^{\prime}, T\right\rangle$ transfer principles must hold. These necessary conditions are not sufficient in general to obtain a factorization of $\mathcal{V}$ (e.g., they are not sufficient for congruence distributive varieties), but the next theorem shows that when $T \subseteq\{\mathbf{1}, \mathbf{2}\}$, then these necessary conditions are sufficient. 
Theorem 2.7. Let $\mathcal{V}$ be a locally finite variety and let $T$ and $T^{\prime}$ be disjoint sets of type labels where typ $\{\mathcal{V}\} \subseteq T \cup T^{\prime}$ and $T \subseteq\{\mathbf{1}, \mathbf{2}\}$. Then $\mathcal{V}=\mathcal{V}_{T} \times \mathcal{V}_{T^{\prime}}$ with typ $\left\{\mathcal{V}_{X}\right\} \subseteq X$ if and only if the following conditions hold:

(1) The $\left\langle T, T^{\prime}\right\rangle$ and $\left\langle T^{\prime}, T\right\rangle$ transfer principles hold and

(2) $\underset{T}{\sim}$ and $\underset{T^{\prime}}{\sim}$ are hereditary.

Proof. We prove only the nontrivial direction, which is that (1) and (2) are sufficient to obtain a decomposition. For $X \in\left\{T, T^{\prime}\right\}$, say that an algebra in $\mathcal{V}$ is locally- $X$ if every finitely generated subalgebra has a congruence lattice with only type $X$ labels. Let $\mathcal{V}_{X}$ denote the collection of all locally- $X$ algebras in $\mathcal{V}$. Using the transfer principles and the hereditariness of $\underset{X}{\widetilde{1}}$, it is not hard to show that $\mathcal{V}_{X}$ is a subvariety of $\mathcal{V}$ of type set $X$ which contains every member of $\mathcal{V}$ of type set $X$. Theorem 2.5 proves that every finitely generated algebra in $\mathcal{V}$ is isomorphic to a product of an algebra from $\mathcal{V}_{T}$ and an algebra from $\mathcal{V}_{T^{\prime}}$. This is enough to prove that $\mathcal{V}=\mathcal{V}_{T} \times \mathcal{V}_{T^{\prime}}$

To augment Theorem 2.7, we reproduce a result (Theorem 3.6) from [1] which gives an easy test for whether $\underset{T}{\sim}$ and $\underset{T^{\prime}}{\sim}$ are hereditary. We state the result with the same hypotheses as Theorem 2.7 even though it is proved under weaker hypotheses in $[1]$.

Theorem 2.8. Let $\mathcal{V}$ be a locally finite variety and let $T$ and $T^{\prime}$ be disjoint sets of type labels where typ $\{\mathcal{V}\} \subseteq T \cup T^{\prime}$. Assume that $\mathcal{V}$ satisfies the $\left\langle T, T^{\prime}\right\rangle$ and $\left\langle T^{\prime}, T\right\rangle$ transfer principles. For $X \in\left\{T, T^{\prime}\right\}$, the congruence $\underset{X}{\sim}$ is hereditary if and only if whenever

(1) $\mathbf{A} \in \mathcal{V}$ is a finite subdirectly irreducible algebra with monolith $\mu$,

(2) $\operatorname{typ}\{\mathbf{A}\} \subseteq X$, and

(3) $\mathbf{B}$ is a subalgebra of $\mathbf{A}$, then typ $\left\{0^{B},\left.\mu\right|_{B}\right\} \subseteq X$.

The following corollary bears the same relationship to Theorem 2.7 that Corollary 2.6 bore to Theorem 2.5 .

Corollary 2.9. Let $\mathcal{V}$ be a locally finite variety and let $T_{1}=\{\mathbf{1}\}, T_{2}=\{\mathbf{2}\}$ and $T_{3} \subseteq\{\mathbf{3}, \mathbf{4}, \mathbf{5}\}$. Assume that typ $\{\mathcal{V}\} \subseteq T_{1} \cup T_{2} \cup T_{3}$. Then $\mathcal{V}=\mathcal{V}_{1} \times \mathcal{V}_{2} \times \mathcal{V}_{3}$ with $\operatorname{typ}\left\{\mathcal{V}_{i}\right\} \subseteq T_{i}$ if and only if the following conditions hold:

(1) The $\left\langle T_{i}, T_{j}\right\rangle$ transfer principles hold for each $i \neq j$, and

(2) $\underset{T_{2}}{\sim}$ and $\underset{T_{3}}{\sim}$ are hereditary.

The only remark necessary is that $\underset{T_{1}}{\sim}$ is always hereditary, since it is the strongly solvable congruence. Therefore, we do not need to check this condition in (2).

\section{REFERENCES}

[1] P. Agliano and K. A. Kearnes, Congruence semimodular varieties I: locally finite varieties, Algebra Universalis 32 (1994), 224-269. MR 95i:08010

[2] R. Freese and R. McKenzie, Commutator Theory for Congruence Modular Varieties, LMS Lecture Notes v. 125, Cambridge University Press, 1987. MR 89c:08006

[3] D. Hobby and R. McKenzie, The Structure of Finite Algebras, Contemporary Mathematics v. 76, American Mathematical Society, 1988. MR 89m:08001 
[4] K. A. Kearnes, A Hamiltonian property for nilpotent algebras, to appear in Algebra Universalis.

[5] R. McKenzie and M. Valeriote, The Structure of Decidable Locally Finite Varieties, Progress in Mathematics v. 79, Birkhäuser, 1989. MR 92j:08001

[6] M. Valeriote, On Decidable Locally Finite Varieties, Ph. D. Dissertation, U. C. Berkeley, 1986.

Department of Mathematical Sciences, University of Arkansas, Fayetteville, ARKANSAS 72701

E-mail address: kearnes@comp.uark.edu 